\title{
QUALIDADE DE VIDA EM IDOSOS PRATICANTES DE ATIVIDADES FÍSICAS
}

DOI: $10.22289 / 2446-922 X . V 3 N 1 A 7$

\author{
Agenísia Maria Viana ${ }^{1}$ \\ Gilmar Antoniassi Junior
}

\section{RESUMO}

A expectativa de vida do brasileiro vem crescendo, com isso surge a necessidade de que as pessoas cuidem mais de sua saúde e isso inclui a prática de atividades físicas regularmente. Entretanto essa é uma preocupação recente, de modo que os idosos nem sempre estiveram atentos para tal necessidade. Diante disso surge o interesse pelo tema deste artigo, que tem como objetivo apontar a importância da prática de atividades físicas por idosos desde que devidamente elaboradas e acompanhadas por um profissional da Educação Física. Para a elaboração deste estudo foi realizada uma pesquisa bibliográfica. Com base nos estudos realizados pode-se concluir que a prática de atividades físicas por idosos traz diversos benefícios, tanto físicos, quanto psicológicos e sociais, sendo um fator de grande relevância para a promoção e manutenção da saúde e qualidade de vida dos mesmos.

Palavras-chave: Saúde. Envelhecimento. Hidroginástica. Caminhada.

\section{ABSTRACT}

The life expectancy of the Brazilian peopleis growing and it arises the need for people to take better care of their health and this includes practicing physical activities regularly. However, this is a recent concern, so the elderly have not always been aware of this need. Faced with this, there is an interest in the theme of this article, which aims to point out the importance of the practice of physical activities by the elderly, since they are properly elaborated and accompanied by a Physical Education professional. For the preparation of this study a bibliographic research has been done. Based on the studies we can conclude that the practice of physical activities by the elderly brings several benefits: physical, psychologica and social, being a factor of great relevance for the promotion and maintenance of health and quality of life.

Keywords: Health. Aging. Hydrogynastic. Walking.

\footnotetext{
1 Endereço eletrônico de contato: agencoro@yahoo.com.br

Recebido em 13/06/2017. Aprovado pelo Conselho Editorial e aceito para publicação em 14/06/2017.
}

Rev. Psicol Saúde e Debate. Jan., 2017:3(1):87-98. 


\section{INTRODUÇÃO}

A expectativa de vida no Brasil e no mundo vem aumentando significativamente o número de pessoas idosas, assim, cada vez mais ativos e lúcidos e em busca de novas possibilidades, os idosos tem buscado práticas atividades físicas. Por possuir uma importante função na melhoria das aptidões físicas e na capacidade funcional, devido à perda de força, diminuição da massa muscular, redução da capacidade cardiovascular, do equilíbrio, flexibilidade, percepção, memória e sensibilidade dos sentidos ${ }^{(1)}$.

Estudo revela que a pratica da atividade física regularmente, associada a uma alimentação saudável, ajuda na prevenção e no controle de doenças específicas da idade, como as doenças coronarianas, articulares, a hipertensão arterial, osteoporose, obesidade sendo benéfica ao organismo e melhorando a qualidade de vida $^{(2)}$.

A realização de atividades regulares é de extrema importância para a saúde física e mental de qualquer indivíduo. No caso dos idosos podem desempenhar uma função de melhoria na sua qualidade de vida, por promover interação com outras pessoas, o bem-estar emocional e físico, aumenta a disposição e alegria ${ }^{(1,2)}$.

Este estudo se justifica pelo diverso aumento da população idosa e da necessidade do bem-estar dos mesmos, feita uma breve analise da literatura percebeu se a necessidade desse estudo por promover uma conscientização da necessidade da interação e da realização de atividades físicas nessa faixa etária.

O presente estudo possui como objetivo apontar a importância prática de atividades físicas por idosos, devidamente elaboradas e acompanhadas por um profissional da Educação Física. 


\section{METODOLOGIA}

O estudo é do tipo qualitativo descritivo de revisão bibliográfica conceitual. Para a revisão do conceito pautou-se na temática qualidade de vida - idoso atividade física. Foram levantados 30 artigos dos quais foram selecionados 19 . Os critérios de busca do material se deram por produções na língua Portuguesa, no período de 1998 a 2016, que estivessem disponíveis na base dados da Scielo, Pubmed, Medline e BVS.

\section{REVISÃO CONCEITUAL}

\subsection{QUALIDADES DE VIDA E ENVELHECIMENTO}

No século XXI têm sido frequentes os debates acerca de Promoção de Saúde entre as pessoas, além de este tema ter se tornado um objeto de pesquisas frequente. Nesse sentido os debates sobre estilos de vida e consequências das atividades físicas na manutenção e recuperação da saúde das pessoas têm ganhado notoriedade. No entanto, ainda existem indivíduos que não valorizam estes benefícios e acabam por não desfrutar destes em suas vidas ${ }^{(3)}$. Nesse contexto muitas pessoas ainda não têm se preocupado com sua qualidade de vida e bemestar, seu foco é a modificação do corpo na busca de uma estética considerada como ideal(4).

As autoridades e a população têm dedicado maior atenção à qualidade de vida, sendo um dos maiores focos da sociedade nos últimos anos. Devido ao fato de ser ainda um conceito novo, de grande abrangência e subjetividade, diversos grupos de estudos iniciaram suas pesquisas sobre o que é e o que abrange este fenômeno(5).

A qualidade de vida e saúde tem passado por melhoras significativas no decorrer dos últimos anos, isto se deve, graças aos avanços econômicos, políticos, sociais e ambientais, bem como a ampliação da conscientização popular, na saúde pública e na medicina ${ }^{(2)}$. 
O termo qualidade de vida recebeu diversas definições no decorrer dos anos, no entanto, pode ser definido em três princípios básicos: capacidade funcional, nível socioeconômico e satisfação. Desse modo, a qualidade de vida e a prática de atividades físicas também estão relacionadas a fatores como: capacidade física, interação social, estado emocional, atividade intelectual, situação econômica e autoproteção de saúde(6).

A conceituação de qualidade de vida se refere ao nível de satisfação dos indivíduos presentes em contexto social amplo, diz respeito a suas necessidades humanas fundamentais, sendo elas, materiais como: água potável, saneamento básico, higiene, saúde, alimentação, educação, entre outros, e imateriais como: segurança, participação, direitos cívicos, entre outros soma-se a esses fatores, suas expectativas sobre a vida e o futuro(2).

O envelhecimento populacional tem sido um grande desafio para a saúde pública atual, isso se deve ao fato de esta ser uma etapa da vida com doenças específicas da idade e que tem ganhado maior notoriedade social. Para favorecer a qualidade de vida dos idosos é importante inserir atividades de promoção à saúde em seu cotidiano e manter hábitos que promovam a melhoria das condições de $v$ ida $^{(5)}$. O idoso enquanto ser social, ainda é visto perante a sociedade de forma secundária, no entanto com o crescimento da expectativa de vida, passar a existir a preocupação em envelhecer com saúde e qualidade, de modo que os atividades físicas se tornam importantes aliados nesta tarefa de buscar saúde e melhor qualidade de vida na terceira idade ${ }^{(6)}$.

O envelhecimento é decorrente de alterações moleculares e celulares, ocasionando perdas funcionais gradativas dos órgãos e do organismo de forma geral. Essa perda começa a ser percebida ao final da fase reprodutiva, ainda que as perdas funcionais do organismo se iniciem bem antes disso, por exemplo, o sistema respiratório e o tecido muscular começam a diminuir suas funções a partir dos 30 $\operatorname{anos}^{(5)}$.

O ser humano passa por modificações fisiológicas, bioquímicas e psicológicas desde o seu nascimento ao chegar velhice acontece ausência gradativa da 
capacidade do organismo em se adaptar às atividades diárias, perda do tônus, elasticidade da pele, atrofiação dos órgãos internos, diminuição do olfato e do paladar, aumenta a quantidade de gordura no organismo; diminui a força muscular(7).

O idoso enfrenta todas as transformações causadas pela idade, além das mudanças sociais, psicológicas e físicas. A sociedade já estabelece que o idoso está fora do mercado de trabalho, algumas famílias os abandonam do convívio familiar, sem falar nas mudanças físicas que podem deixá-los debilitados, algumas dessas alterações são: a perda de massa óssea, a diminuição dos músculos, da flexibilidade, além da possibilidade de sofrerem mais de hipertensão, arteriosclerose, artrose, artrite, osteoporose, entre outras doenças ${ }^{(6)}$.

Os efeitos associados à falta de atividades físicas levam o idoso, a uma condição de degeneração progressiva de suas capacidades físicas ocasionando uma diminuição no rendimento físico, nas habilidades motoras, na concentração, nas capacidades de coordenação e reação, causando nele uma sensação de autodesvalorização, insegurança, impassibilidade, desmotivação, isolamento social e a solidão(8).

\subsection{A IMPORTANCIA DAS ATIVIDADES FÍSICAS PARA O IDOSO}

A atividade física e a qualidade de vida, sempre foram uma preocupação do ser humano, mas, atualmente, a busca constante de uma vida saudável tem se tornado um compromisso pessoal, desenvolvida juntamente com o bem-estar, associado às condições do modo de vida, como: saúde, moradia, educação, lazer, transporte, liberdade, trabalho e autoestima ${ }^{(6)}$.

A atividade física pode ser definida como qualquer movimento corporal realizado pela musculatura que tenha como resultado um gasto energético além dos níveis do corpo quando em repouso. A prática regular de atividades físicas proporciona benefícios em todos os aspectos do organismo(5).

Os benefícios da atividade física para a saúde são diversos proporcionando um melhor funcionamento corporal, independência; redução no risco de morte por doenças cardiovasculares; controle da pressão arterial; recuperação na postura e no equilíbrio; controle do peso corporal; utilização mais eficaz da glicose pelo Rev. Psicol Saúde e Debate. Jan., 2017:3(1):87-98. 
organismo; melhoria no retorno venoso; prevenção a cãibras, função intestinal regulada; melhora o sistema imunológico; promove a qualidade do sono; amplia as relações sociais; reduz a ansiedade; o estresse, as alterações de humor e melhora a

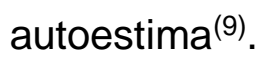

A atividade física interfere de forma positiva na saúde física e mental dos indivíduos, sendo de grande importância em todas as fases da vida, desde criança até a velhice. Quando realizada de maneira regular, é uma das bases fundamentais para a manutenção da saúde, juntamente com uma alimentação balanceada e um estado emocional equilibrado(6).

Ao praticar exercícios regularmente é uma maneira efetiva para diminuir e/ou prevenir a decadência decorrente do processo de envelhecimento, modificando a condição de incapacidade para o cumprimento das atividades da vida $\operatorname{cotidiana}^{(8)}$. Para tentar amenizar essas perdas decorrentes da idade, a prática de atividades físicas é uma grande aliada, pois favorece a liberação de substâncias que ativam sistemas corporais, estimula as funções metabólicas e vitais do corpo contribuindo para a qualidade de vida do idoso(5).

As atividades físicas regulares promovem a melhoria da força, da massa muscular bem como a flexibilidade das articulações, atuando também como forma de prevenção às doenças neurológicas, tais como o mal de Alzheimer e a esclerose múltipla ${ }^{(10)}$. A prática de atividade física é uma maneira de promover a melhoria da autonomia do idoso e consequentemente sua qualidade de vida para que ele consiga executar até mesmo as tarefas simples do dia a dia com independência como realizar sua higiene pessoal, ter força e equilíbrio para caminhar, descer de um ônibus, subir e descer escadas e até mesmo dançar ${ }^{(6)}$.

$\mathrm{O}$ ato de praticar atividades físicas combate o sedentarismo, colabora significativamente para a manutenção das capacidades físicas do idoso. Isso não significa que o processo de envelhecimento seja interrompido, entretanto reduz os danos decorrentes da idade, de modo a manter as habilidades do idoso por mais tempo, dando-Ihe autonomia, interferindo diretamente em sua qualidade de vida 
assim sendo que as atividades físicas ou práticas preventivas sejam elaboradas por profissionais que compreendam melhor essa fase da vida da população idosa(8).

Na Atualidade o mercado de trabalho tem se tornado cada vez mais exigente e com grande especificidade sendo que os profissionais das diversas áreas têm tido que desenvolver ainda mais suas competências para se inserir e mante-se nesse $\operatorname{mercado}^{(11)}$.

O mercado de atuação do profissional de Educação Física é amplo, tendo várias colocações na sociedade, as academias de atividades esportivas podem ser avaliadas como a opção mais interessante em meio às áreas de atuação desses profissionais, sendo a preferência de vários deles, sobretudo dos recémformados $^{(12)}$. O foco de atuação do profissional de educação física tem em vista prevenir, promover, proteger e reabilitar a saúde no contexto social de uma população ou indivíduo. O educador físico precisa estar habilitado para trabalhar em equipe multiprofissional, dentro das atividades de gestão e para lidar com políticas de saúde, bem como para diagnosticar, planejar e intervir no campo das práticas corporais e atividades físicas ${ }^{(13)}$.

Uma das funções do profissional de Educação Física é adequar as atividades físicas de modo que elas sejam realizadas da forma correta. Para que um exercício seja eficaz, o indivíduo não precisa ficar exausto, executando movimentos forçados de intensidade elevada e longa duração, é importante que o profissional tome cuidado com fatores como o aumento da frequência cardíaca e da pressão arterial $^{(14)}$.

O profissional de Educação Física tem por função prescrever, nortear e acompanhar as pessoas na realização de atividades físicas ou desportivas ${ }^{(11)}$. É comum que as pessoas comecem a realizar atividades físicas devido a recomendações médicas, no entanto, o profissional de educação física que irá supervisionar a realização dessas atividades, de modo que os resultados na medicina sejam alcançados juntamente com as atividades físicas, sendo o responsável pela orientação técnica, tática e física(14). 
O profissional de Educação Física precisa motivar o aluno e durante atividade física, fazendo com que este sinta prazer, alegria e satisfação ao realizar a atividade, mostrando através dos exercícios a importância de praticá-los em prol de uma melhor qualidade de vida, seja nos aspectos físicos, psicológicos ou sociais ${ }^{(15)}$.

São várias as atividades definidas como atividades físicas, que podem ser utilizadas para viabilizar a qualidade de vida de idosos. Dentre elas podem ser citadas: caminhar a pé, jogar futebol, andar de bicicleta, dentre outras e quando são praticadas rotineiramente tendem a deixar o indivíduo com mais disposição para a vida e menos propenso a doenças crônicas ${ }^{(3)}$.

\subsection{ANÁLISES SOBRE QUALIDADE DE VIDA EM IDOSOS PRATICANTES DE ATIVIDADES FÍSICAS}

A prática regular de atividade física de forma preventiva preserva a qualidade de vida dos idosos além de melhorar a capacidade respiratória, força muscular, memória recente, reserva cardíaca, influencia suas habilidades sociais, predisposição física e capacidade funcional(1). Para iniciar qualquer tipo de atividade física é necessária realizar uma avaliação médica e alguns exames clínicos para avaliar as condições cardiovasculares ${ }^{(16)}$. Dentre as atividades físicas que são realizadas com mais frequência pelos idosos será feita aqui a analise da hidroginástica e da caminhada.

A hidroginástica é uma forma de condicionamento físico constituída de atividades aquáticas específicas, baseadas no aproveitamento da resistência da água como sobre carga. Desde 460-375 a.C. a água já era utilizada com fins terapêuticos com finalidades recreativas e curativas. A hidroginástica começou a ser desenvolvida de maneira sistemática no início do século $X X I$ nos spas ingleses ${ }^{(17)}$.

A hidroginástica na terceira idade como uma atividade física tem predominância do gênero feminino por existirem mais mulheres nessa faixa etária do que homens, sendo também o fator de conscientização com o objetivo de conservar a saúde através da prática da atividade física maior nas mulheres. Propicia uma qualidade de vida ao idoso por meio do fortalecimento da musculatura, a capacidade de rendimento do coração, da circulação, dos pulmões, elasticidade dos músculos, 
tendões e ligamentos, a velocidade dos processos metabólicos possibilita a prevenção e tratamento das doenças próprias do idoso(18).

A hidroginástica tem o efeito de reduzir gordura corporal proporcionando uma massagem no local em movimento tem como fator principal a diminuição do impacto e da incidência da atuação da gravidade sobre o corpo como efeitos terapêuticos de alívio da dor e relaxamento, fortalecimento da musculatura sem provocar lesão nas articulações, aumenta a circulação estimula a autoestima, alegria auxiliando na melhora das práticas cotidianas pode-se utilizar de aparelhos com objetivo de aumentar a resistência do trabalho com a água(17).

Muitos escolhem a atividade por recomendação médica com restrições que devem ser consideradas na prescrição e na escolha dos objetivos. As atividades devem buscar amplitude, coordenação e a atuação dos grandes grupamentos musculares os movimentos devem primar pelo equilíbrio e simplicidade ${ }^{(18)}$.

A caminhada é uma atividade física democrática aberta a qualquer pessoa. 0 movimento básico é a marcha, de baixa complexidade de execução que pode ser praticada sem custos e que pode ser realizada em qualquer lugar, seja nas ruas, nos parques, na praia, no campo, em pistas atléticas, ginásios ou em área interna de condomínios, qualquer lugar é adequado para uma boa caminhada observando as condições das pistas para que não haja riscos de quedas, lesões e desconforto recomenda se a pratica da caminhada de três a seis vezes por semana, com intensidade leve ou moderada, sessões de 30 a 60 minutos de duração antecedidos por aquecimento e finalizados com um alongamento muscular(19).

Caminhar é a atividade física que contribui com a melhora na função cardiovascular, o nível de condicionamento físico; na perda de peso e, evitando quedas e auxiliando na agilidade; reduz pressão sanguínea, os níveis de colesterol no sangue, osteoporose, diabetes e o estresse, além de melhorar a autoestima, evitar a depressão, auxilia no convívio social ${ }^{(16)}$.

Os benefícios da caminhada para o idoso, não se restringe somente as melhorias físicas registram-se também no âmbito psicológico, colaborando com estados de humor, raiva, tensão, depressão, possibilitando vitalidade. Caminhar é 
uma prática simples e conveniente para a promoção da atividade física regular utilizada pelos indivíduos nos tempos livres, de lazer para melhor qualidade de vida(19). $^{(19)}$

\section{CONCLUSÃO}

Manter hábitos saudáveis no decorrer da vida desempenha uma ação primordial para um envelhecimento saudável. Uma rotina de cuidados com a alimentação, evitar o consumo de cigarro e de álcool principalmente a prática de atividade física, são condições que colaboram para que a pessoa tenha uma melhor qualidade de vida na velhice.

Os benefícios fundamentais decorrentes da prática de atividades físicas regulares para o idoso têm sido amplamente estudados, graças ao conhecimento de que manter um estilo de vida ativo gera a manutenção da capacidade funcional por um período maior de tempo e mantem sua qualidade de vida.

Com base na pesquisa bibliográfica realizada, perceber se que a prática de atividades físicas representa uma melhora significativa nos problemas de saúde, diminuindo as dores corporais, problemas com depressão, favorecendo a autonomia na realização de atividades cotidianas, além de promover bem-estar físico e mental. Na terceira idade, a estabilidade emocional é um dos fatores que requer atenção, sendo que a atividade física tem por objetivo melhorar a qualidade de vida como um todo isso inclui as questões físicas, emocionais e sociais.

Neste contexto faz-se necessário ressaltar a importância do profissional de Educação Física para a elaboração de uma rotina de atividades físicas adequadas a cada indivíduo, de acordo com suas necessidades e condições físicas, motivando-as para a sua realização considerando que tal pratica pode ocorrer por recomendação médica, no entanto, o idoso não se sente motivado para realizá-las.

Portanto, pode-se concluir que as atividades de hidroginástica e caminhada viabiliza aptidão física, tais como força, flexibilidade e equilíbrio, que são aspectos importantes para a realização das atividades cotidianas e favorecem a qualidade de vida aspecto de suma importância no contexto social atual. 


\section{REFERÊNCIAS}

1. Ferretti F, Beskow GCT, Slavieno RC, Ribeiro CG. Análise da qualidade de vida em idosos praticantes e não praticantes de exercício físico regular. Estud interdiscipl envelhec. 2015;20(3): 729-743.

2. Diniz F, Neves MP, Hermones R, Freitas VL. Promoção de saúde e qualidade de vida: uma revisão bibliográfica. Acta Cient. 2010;2(2):53-63.

3. Silva LAM, Silva R M. Efeitos da Atividade Física na Promoção da Saúde: percepção de jogadores de futebol do município de Coromandel /MG. Acta Cient. 2011;3(3):149-160.

4. Brandão FR, Antoniassi Júnior G. O uso de substâncias nocivas associadas ao comportamento de risco do praticante de atividade física. Rev Psicol Saúde Debate 2015;1(1):53-64.

5. Ferreira T KA, Pires VA, Teles Netto. Atividade física na velhice: avaliação de um grupo de idosas sobre seus benefícios. Rev Enferm Integ. 2015;8(1):1303-1313.

6. Dutra JS. Atividade física e qualidade de vida no grupo de idosos. [Monografia] Criciúma: Universidade do Extremo Sul Catarinense - UNESC; 2006.

7. Gomes AM. Ações do enfermeiro na assistência ao idoso portador de demência de Alzheimer. [TCC] Vitória: Faculdade Católica Salesiana Do Espírito Santo; 2013.

8. Araújo LF. Benefícios da atividade física segundo os idosos hipertensos e diabéticos do programa de saúde da família. [Monografia] Porto Velho: Universidade Federal de Rondônia; 2008.

9. Brasil. Ministério da Saúde. Departamento de Ações Programáticas e Estratégicas. Secretaria de Atenção à Saúde. Estatuto do Idoso. 3aㅡ ed. Brasília, DF, 2007.

10. Rocha ECA. Prática de Atividades Físicas X Saúde do Idoso. [Monografia] Feira de Santana: Faculdade de Tecnologia e Ciências; 2009.

11. Ramos MM, Bonvicin CR. Estudo das Competências: profissionais da saúde. Psicol Saúde Debate. 2016;2(Edição Especial):28-40. 
12. Oliveira AL, Silva MP. O profissional de educação física e a responsabilidade legal que o cerca: fundamentos para uma discussão. In: Anais do IX Simpósio Internacional Processo Civilizador: Tecnologia e Civilização; 24-26 de out 2005; Ponta Grossa, BR. Ponta Grossa: UTRPR, 2005.

13. Scabar TG, Pelicioni AF, Pelicioni MCF. Atuação do profissional de Educação Física no Sistema Único de Saúde: uma análise a partir da Política Nacional de Promoção da Saúde e das Diretrizes do Núcleo de Apoio à Saúde da Família - NASF. J Health Sci Inst. 2012;30(4):411-8.

14. Civinski C, Montibeller A, Braz ALO. A importância do exercício físico no envelhecimento. Rev UNIFEBE. 2011;1(9):163-175.

15. Silva WA. Atividade Física e terceira idade: um estudo de caso sobre as atividades oferecidas pelo Grupo Viver Feliz, do Munícipio de Águas Lindas de Goiás. [ Monografia] Ceilândia: Universidade de Brasília; 2012.

16. Melo DG. Benefícios da prática da caminhada para os idosos do grupo "terceira idade" de Cavalcante-GO. [Monografia] Alto Paraíso: Universidade de Brasília Faculdade de Educação Física Programa Universidade Aberta do Brasil - Pólo Alto Paraíso - GO; 2012.

17. Marciano GG, Vasconcelos APSL. Os benefícios da hidroginástica na vida diária do idoso. Rev Eletronica Fac Metod Granbery. 2008;(4).

18. Paula KC, Paula DC. Hidroginástica na terceira idade. Rev Bras Med Esporte. 1998;4(1):24-27.

19. Cavalcante I, Nobre J, Paiva P, Borragine SOF. Contribuições da caminhada para melhor qualidade de vida de idosos. EFDeportes.com 2011;16(157):1-1.

Rev. Psicol Saúde e Debate. Jan., 2017:3(1):87-98. 